Article

\title{
Functional Characterization of Selected Universal Stress Protein from Salvia miltiorrhiza (SmUSP) in Escherichia coli
}

\author{
Xiao-Fan Wang, Jiao Su, Na Yang, Hui Zhang, Xiao-Yan Cao * and Jie-Fang Kang * \\ Key Laboratory of the Ministry of Education for Medicinal Resources and Natural Pharmaceutical Chemistry, \\ National Engineering Laboratory for Resource Development of Endangered Crude Drugs in Northwest of China, \\ Shaanxi Normal University, Xi'an 710062, China; fanfan9138@163.com (X.-F.W.); sujiao@snnu.edu.cn (J.S.); \\ m18829222318@163.com (N.Y.); 201503829@snnu.edu.cn (H.Z.) \\ * Correspondence: caoxiaoyan@snnu.edu.cn (X.-Y.C.); kangjiefang@snnu.edu.cn (J.-F.K.); \\ Tel.: +86-29-8531-0680 (X.-Y.C.); +86-29-8531-0260 (J.-F.K.); Fax: +86-29-8531-0280 (X.-Y.C. \& J.-F.K.)
}

Received: 1 July 2017; Accepted: 5 September 2017; Published: 8 September 2017

\begin{abstract}
The multigene universal stress protein (USP) family is evolutionarily conserved. Members play indispensable roles in plant tolerance to abiotic stresses. Although relatively well-characterized in model plants, such as Arabidopsis thaliana and Oryza sativa, this family has not been investigated in Salvia miltiorrhiza, an important herbal plant for which yields can be limited by various abiotic stresses. Here, we identified 32 USP family members in the S. miltiorrhiza genome, and used phylogenetic analysis to sort these SmUSPs into four groups. Groups A and B belong to the ATP-binding class whereas Groups $C$ and D are in the non-ATP-binding class. Motif analysis and multiple sequence alignment hinted that members of group A and B were able to bind ATP. Our qRT-PCR data from different tissues/organs and under salt and heat stresses provided an overall expression pattern for those genes. Three SmUSPs (SmUSP1, SmUSP8, and SmUSP27) were cloned from S. miltiorrhiza and functionally characterized in Escherichia coli. Compared with the control cells, those that expressed SmUSPs exhibited enhanced tolerance to salt, heat, and a combination of the two. This suggested that the protein has a protective role in cells when exposed to single-stress and multiple-stress conditions. Our findings provide valuable information that helps improve our understanding of the evolutionary and functional conservation and diversity associated with the USP gene family in S. miltiorrhiza.
\end{abstract}

Keywords: universal stress protein (USP); gene family; Salvia miltiorrhiza; Escherichia coli; abiotic stress

\section{Introduction}

Abiotic stresses, such as drought, salinity, and extreme temperatures, are limiting factors for normal plant growth. Excessive salinity interrupts homeostasis in water potential and ion distribution at both the cellular and whole-plant level [1]. This results in molecular damage, arrested development, and even mortality [2]. High temperatures can cause irreversible damage, including protein denaturation, aggregation, and degradation [3], as well as inhibition of protein synthesis and inactivation of enzymes. Extreme temperatures can also increase the fluidity of membrane lipids, leading to a loss of membrane integrity [4]. Field environments are very different from the controlled conditions used in laboratory studies, and outdoor experiments often involve exposing plants simultaneously to more than one type of abiotic and/or biotic stress, e.g., salinity plus heat, a combination that is probably the major environmental factor that restricts growth and yields in the 21st Century [5]. 
The family of universal stress protein genes (USPs) appears to play a positive role in the abiotic-stress response, with members encoding proteins that contain highly conserved residues of the Universal Stress Protein A domain (UspA domain; Pfam Accession Number PF00582). These genes are widely distributed across most living organisms, including bacteria, archaea, fungi, protozoa, plants, and mammals [6-8]. The first USP was discovered in a bacterium, and was initially named C13.5 protein because of its migration on a two-dimensional Isoelectric Focusing-PAGE gel. That name was later changed to USP to reflect its ability to respond to diverse stresses. The label UspA was quickly and extensively acknowledged, and now represents an orthologous group of proteins, the UspA superfamily [9]. Six USP genes in Escherichia coli have different functions linked to motility, adhesion, and resistance to oxidative stress [10]. Homologs of USPs are ubiquitous in plants and encoded by gene families. They include 44 Arabidopsis proteins that are similar to the UspA domains in bacteria as well as 16 putative USP genes in barley (Hordeum vulgare) [6,11]. This gene family comprises two classes: ATP-binding and non-ATP-binding [12].

Although USP genes have been characterized in diverse plant species, including barley, A. thaliana (hereafter, Arabidopsis), Oryza sativa, Gossypium arboreum, Astragalus sinicus, Vicia faba, Solanum pennellii, and viridiplantae, their functions remain largely unknown [6,11,13-17]. It appears that USP improves the rate of cell survival during prolonged exposure to stress agents, and may endow plants with wide-ranging stress tolerance [18]. For example, SpUSP in S. pennellii reduces the stomatal aperture to protect plants from the effects of drought [10]. Among the Arabidopsis USP genes, At5g54430 and At4g27320 are phosphorylated in response to microbial elicitation of the cells [17]. At3g53990 exhibits a chaperone function and is significantly induced by heat, $\mathrm{H}_{2} \mathrm{O}_{2}$, and drought treatments [19]. Another Arabidopsis USP gene, At3g62550, encodes an ATP-binding motif and has been shown to be up-regulated in a drought microarray dataset [17].

Salvia miltiorrhiza (family Labiatae) is an important herbal plant. Its dried roots, also known as 'Danshen', are widely used in modern and traditional Chinese medicine (TCM) for treating cardiovascular/cerebrovascular diseases and various symptoms of inflammation [20-22]. This species is emerging as a model plant for TCM studies because of its relatively small genome $(600 \mathrm{Mb})$, short life cycle, minimal growth requirements, and significant medicinal value [23]. Although the functions of numerous genes from S. miltiorrhiza have been reported, information about the SmUSP gene family is lacking. The genomic database of $S$. miltiorrhiza has been published online at http:/ /www.ndctcm.org/shujukujieshao/2015-04-23/27.html [24]. Heterologous in vivo expression in E. coli is an approach now available for functional characterization of stress-responsive genes [25]. Here, we identified USP members from the genomic database of S. miltiorrhiza. By integrating the results from phylogenetic analysis and investigations of protein motif structures, gene expression, and heterologous expression in E. coli, we were able to determine the evolutionary and functional conservation and diversity of this gene family in S. miltiorrhiza. Our findings provide a genetic resource for improving abiotic stress tolerance by S. miltiorrhiza through genetic engineering technologies.

\section{Materials and Methods}

\subsection{Identification of USP Genes from the Salvia miltiorrhiza Genomic Database}

Using the conserved domain search service of the NCBI database (www.ncbi.nlm.nih.gov/ Structure/cdd/wrpsb.cgi), we found 44 Arabidopsis protein sequences with similarity to the UspA domains of bacteria [6], and obtained 26 UspA domains. Those conserved domain sequences were used to search, with TBLASTN (e-value $<10^{-10}$ ), for the sequences of USPs in the Salvia miltiorrhiza genomic database [24]. All of the identified candidates were analyzed using the protein family database (Pfam; http:/ / pfam.sanger.ac.uk/) to confirm the presence of UspA domains in their protein structure. 


\subsection{Bioinformatics Analysis, Phylogenetic Analysis, and Multiple Sequence Alignment}

The molecular weights, theoretical pI values, and number of amino acids for the 32 SmUSPs were predicted using the Compute pI/Mw tool on the ExPASy server (http:/ /web.expasy.org/compute_ pi/) [26]. Their conserved protein motifs were examined with MEME v4.11.2 software (http://memesuite.org/tools/meme), which revealed 10 different motifs, and minimum motif and maximum motif windows set to 6 and 50, respectively. A signal peptide analysis was conducted using the TargetP algorithm (TargetP: http:/ / www.cbs.dtu.dk/services/TargetP/). Finally, the exon-intron structures of SmUSPs were determined via GSDS 2.0 (http://gsds.cbi.pku.edu.cn/), comparing the full-length cDNA sequences to the genomic sequences.

We used MEGA 6 software to construct a phylogenetic tree by the Neighbor Joining method, according to the following parameters: P-distance model, pairwise deletions, and 1000-replicates bootstrap. Multiple sequence alignments of 21 SmUSPs and the USP sequence of Methanococcus jannaschii MJ0577 (1MJHA; NCBI protein GI: 5107801), were generated by DNAMAN v6.0.3.99 software, with default parameters.

\subsection{Plant Growth and Stress Treatments}

For expression profiling, we placed 2-month-old, uniformly developed S. miltiorrhiza plants in a greenhouse (16-h photoperiod, $25^{\circ} \mathrm{C}$ ). Salt stress was induced by watering the plants with a $150 \mathrm{mM}$ $\mathrm{NaCl}$ solution, heat-stress conditions were imposed by transferring the plants to a growth chamber and holding them at $38^{\circ} \mathrm{C}$. For the combined salt/heat treatment, the plants were watered with $150 \mathrm{mM}$ $\mathrm{NaCl}$ solution, then transferred to the chamber and held at $38^{\circ} \mathrm{C}$. After $24 \mathrm{~h}$, leaves were collected from different plants (three biological replicates), immediately frozen in liquid nitrogen, and stored at $-80^{\circ} \mathrm{C}$. For the control, plants continued to receive normal irrigation in the greenhouse, under the conditions described above, throughout the experimental period. Their leaves were also collected and stored at $-80^{\circ} \mathrm{C}$.

\subsection{Promoter Sequence Analysis, RNA Isolation, and Quantitative RT-PCR Analysis}

The 2-kb genomic sequence upstream of the 5'-UTR of each SmUSP was obtained for putative cis-element analysis, using the PlantCARE database (http:/ / bioinformatics.psb.ugent.be/webtools / plantcare/html/). Quantitative RT-PCR (qRT-PCR) analysis was performed to monitor the expression levels of SmUSPs in the roots, stems, leaves, flowers, and seeds, as well as under various stress treatments. The gene-specific primers are listed in Table S1. Total RNA was isolated using an RNA rapid extraction kit (Xi'an Chunfeng, Xi'an, China) according to the manufacturer's instructions. First-strand cDNA was synthesized with reverse transcriptase (Takara, Beijing, China) by following the manufacturer's protocol, and qRT-PCR was conducted on a LightCycler 96 system (Roche, Basel, Switzerland). The reaction mixture contained $10 \mu \mathrm{L}$ of $2 \times$ SYBR Premix Ex Taq II (Takara, Beijing, China), $500 \mathrm{nM}$ each of sense and antisense primers, $20 \mathrm{ng}$ of first-strand cDNA. Initial thermal-cycling at $95{ }^{\circ} \mathrm{C}$ for $30 \mathrm{~s}$ was followed by 45 cycles of $95^{\circ} \mathrm{C}$ for $10 \mathrm{~s}$ and $60^{\circ} \mathrm{C}$ for $30 \mathrm{~s}$. Ubiquitin (GenBank Accession No. JF760206.1) served as the internal control. Relative gene expression levels were analyzed according to the comparative $2^{-\Delta \Delta C(\mathrm{~T})}$ method [27].

\subsection{Molecular Cloning and Construction of Escherichia coli Strains Expressing Universal Stress Proteins}

Full-length opening read frames of SmUSP1, SmUSP8, and SmUSP27 were cloned using high-fidelity ARHS DNA polymerase (Takara), and restriction sites were added to the primers (Table S2). The PCR conditions consisted of an initial denaturation at $98^{\circ} \mathrm{C}$ for $1 \mathrm{~min}$; then 30 cycles at $98^{\circ} \mathrm{C}$ for $10 \mathrm{~s}$, $55{ }^{\circ} \mathrm{C} / 58{ }^{\circ} \mathrm{C} / 59{ }^{\circ} \mathrm{C}$ for $25 \mathrm{~s}$, and $72{ }^{\circ} \mathrm{C}$ for $60 \mathrm{~s}$; followed by a final extension at $72{ }^{\circ} \mathrm{C}$ for $10 \mathrm{~min}$. The PCR products were digested with SacI and XhoI/BamHI and HindIII/BamHI and XhoI (New England BioLabs, or NEB, Ipswich, MA, USA). Afterward, the fragments of SmUSPs, with sticky SacI, 
XhoI/BamHI, HindIII/BamHI, and XhoI (NEB), were inserted into pET28a vectors and sequenced to create recombinant plasmids pET28a-SmUSPs.

These plasmids plus the empty vector pET28a were transformed into E. coli BL21 strain. Cells carrying either pET28a-USPs or pET28a were cultured overnight at $37{ }^{\circ} \mathrm{C}$ in Luria-Bertani (LB) liquid media containing $50 \mathrm{mg} \mathrm{L}^{-1}$ kanamycin (kan). They were then diluted 1000 times with fresh LB liquid media containing $50 \mathrm{mg} \mathrm{L}^{-1} \mathrm{kan}$ and incubated continually until the cells reached the mid-log phase $\left(\mathrm{OD}_{600 \mathrm{~nm}}=0.6\right)$. Isopropyl b-D-thiogalactopyranoside (IPTG) was added to a final concentration of $1 \mathrm{mM}$ and the cells were cultured for another $6 \mathrm{~h}$. They were then harvested by centrifugation, re-suspended in LB liquid media, and mixed with $5 \times$ SDS loading buffer (Zhuangmeng Biology, Beijing, China). After being held in a $100{ }^{\circ} \mathrm{C}$ water bath for $5 \mathrm{~min}$, the mixture was used for SDS-PAGE analysis. For western blot, HIS-Tag mouse monoclonal antibody was used for detection (Beijing Biodragon Immunotechnologies Co., Ltd., Beijing, China) along with HRP Goat anti-mouse Ig (H + L) (Abbkine Scientific Co., Ltd., California, USA). The immunoreactive bands were detected by chemiluminescence and pictured with the ChemiDoc ${ }^{\mathrm{TM}}$ MP Imaging System (BIO-RAD, Hercules, CA, USA).

\subsection{Salt, Heat, and Combined Treatments with Transformed E. coli Cells}

Cell cultures of E. coli were grown as described above. After IPTG induction for $6 \mathrm{~h}$, the $\mathrm{OD}_{600}$ was determined for each sample so that they could be equilibrated to the same value. Afterward, each sample was diluted 10,100, or 1000 times. For studying their tolerance to salinity, $7-\mu \mathrm{L}$ samples from those different dilutions were spotted onto LB solid media containing $50 \mathrm{mg} \mathrm{L}^{-1} \mathrm{kan}$ plus $200 \mathrm{mM}$ $\mathrm{NaCl}$. The plates were incubated upside-down overnight at $37^{\circ} \mathrm{C}$. Samples with the same $\mathrm{OD}_{600}$ value were then inoculated into an LB liquid medium (1:200 ratio) containing $50 \mathrm{mg} \mathrm{L}^{-1} \mathrm{kan}$ plus $200 \mathrm{mM}$ $\mathrm{NaCl}$ and measurements were made at 2-h intervals to produce a growth curve.

For evaluating their degree of heat tolerance, samples of the different dilutions were exposed to $50{ }^{\circ} \mathrm{C}$ for $60 \mathrm{~min}$ before $7-\mu \mathrm{L}$ aliquots were spotted on $\mathrm{LB}$ media containing $50 \mathrm{mg} \mathrm{L}^{-1} \mathrm{kan}$. The plates were incubated upside-down overnight at $37^{\circ} \mathrm{C}$. Samples with the same $\mathrm{OD}_{600}$ value were exposed to $50{ }^{\circ} \mathrm{C}$ for $30 \mathrm{~min}$ before being inoculated into an LB liquid medium (1:200 ratio) containing $50 \mathrm{mg} \mathrm{L}^{-1} \mathrm{kan}$. Measurements were made at 2-h intervals to produce a growth curve.

For studying their tolerance to the combination of salt and heat stressors, the same procedures as for evaluating heat tolerance were utilized, except that the LB medium contained both $50 \mathrm{mg} \mathrm{L}^{-1} \mathrm{kan}$ and $200 \mathrm{mM} \mathrm{NaCl}$.

\subsection{Statistical Analysis}

All data were presented as the means \pm Standard Deviation (SD) of at least three replicates. Statistical analysis was performed with SPSS 17.0 software (IBM, Armonk, NY, USA). Analysis of variance (ANOVA) was followed by Tukey's pairwise comparison tests. Mean values were considered significantly different at $p<0.05$.

\section{Results}

\subsection{Gene Identification, Features of Sequences, and Phylogenetic Analysis}

We identified 32 SmUSP genes that contain the UspA domain. They were named SmUSP1 through SmUSP32, based on their annotated gene IDs (NCBI Accession Number: MF614040 to MF614071). Salvia miltiorrhiza has fewer USPs than Arabidopsis. The genomic DNA of the former group ranges in length from $362 \mathrm{bp}$ (SmUSP11) to $5478 \mathrm{bp}$ (SmUSP5), while the cDNAs are $279 \mathrm{bp}$ (SmUSP11) to $2274 \mathrm{bp}$ (SmUSP3) long (Table S3). The molecular weights of the predicted SmUSPs vary from 10,361.16 Da (SmUSP11) to 83,958.85 Da (SmUSP3), while the theoretical isoelectric points are predicted to range from 4.87 (SmUSP15) to 11.16 (SmUSP30). 
A phylogenetic analysis based on full-length protein sequences revealed that these 32 SmUSPs can be arranged into four groups together with the 26 Arabidopsis USPs that also contain the UspA domain, thereby confirming that all belong to the same gene family (Figure 1). Subfamily A has the most, i.e., 20 SmUSPs while subfamily B has only one member, SmUSP10.

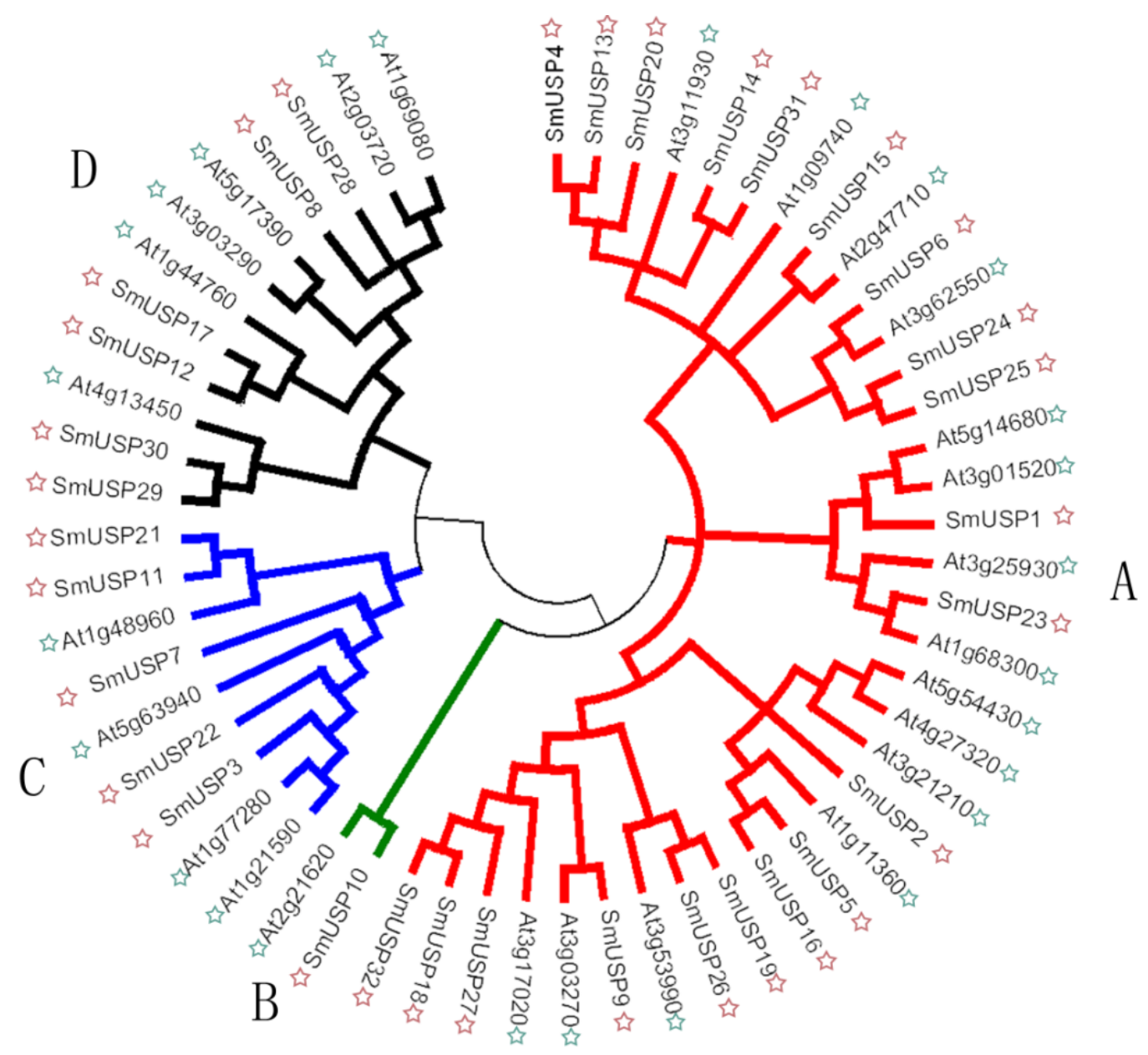

Figure 1. Phylogenetic relationship of Salvia miltiorrhiza and Arabidopsis thaliana universal stress protein genes (USPs) that contain UspA domain. USP groups are distinguished by color of branch. Green stars, USPs from Arabidopsis; red stars, USPs from S. miltiorrhiza.

\subsection{Conserved Motifs, Multiple Sequence Alignments, Genetic Structures, and Analysis of Subcellular Locations}

We identified 10 motifs that contain 6 to 50 residues (Figures 2A and S1). The motif patterns are conserved among the 32 SmUSPs and members within each group have similar organizations. For example, Motif 2 is shared by 27 SmUSPs; Motif 3, by 24 SmUSPs. Motif 1 contains the ATP binding motif (G-2X-G-9X-G[S/T]), which is distributed only in subfamilies A and B (Figure 2B) [28]; Motif 10 is found only in subfamily C; Motif 4 is shared by six members (SmUSP9, 18, 19, 26, 27, and 32) in subfamily A; Motif 6 is shared by seven members (SmUSP4, 13, 14, 20, 24, 25, and 31) in subfamily A; and Motif 5 is shared by three members (SmUSP3, 7, and 22) in subfamily C. 
(A)

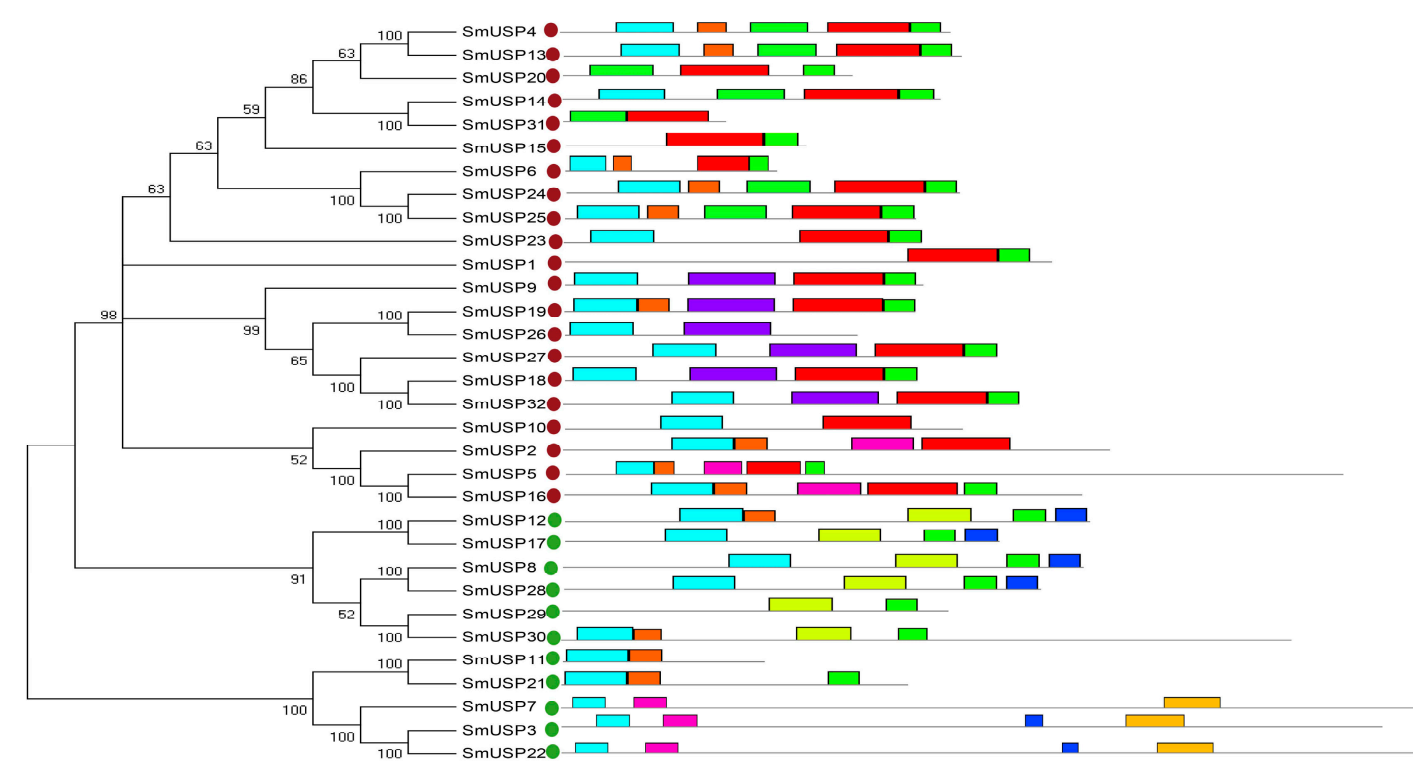

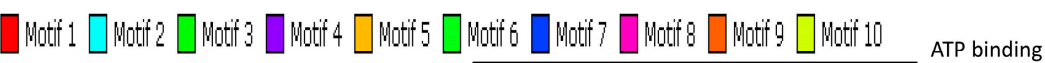

(B)

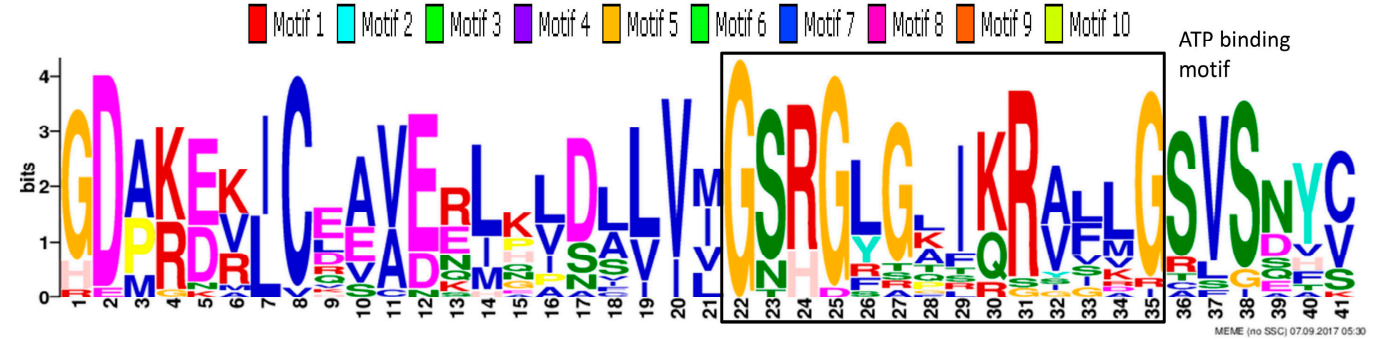

Figure 2. Conserved motifs of SmUSP. (A) Conserved motifs and phylogenetic tree of 32 SmUSPs. Red spots, members of ATP-binding class; green spots, members of non-ATP-binding class; (B) ATP-binding motif (G-2X-G-9X-G[S/T]) is marked.

Motif 1 is distributed only in subfamilies A and B, which indicates that all 21 members have the ATP-binding structure. To confirm the sequence similarity of those 21 SmUSPs to the USP sequence of M. jannaschii MJ0577 (1MJHA), we performed a multiple sequence alignment (Figure 3).

Analysis of the exon-intron organization structures of all SmUSPs revealed that they contain one to 10 exons, while two genes (in subfamily A) lack introns (Figure 4). An excess of symmetric exons and phase- 0 introns is likely to facilitate exon-shuffling, recombinational fusion, and an exchange of protein domains $[29,30]$. Among the 100 introns found in these SmUSPs, 40 are phase 0, 28 are phase 1, and 32 are phase 2. Our analysis of the SmUSP structures demonstrated that these genes are relatively well-conserved.

We then predicted subcellular distributions and found that the 21 SmUSPs are localized in the cytosol or nucleus (in Table S3, "other" means that no signal peptide was detected). Our results showed that SmUSP8, SmUSP15, SmUSP27, and SmUSP28 are probably targeted to mitochondria; SmUSP2, SmUSP5, SmUSP20, and SmUSP29 are probably targeted to chloroplasts; and SmUSP2 and SmUSP16 are exclusively targeted to a secretory pathway. 


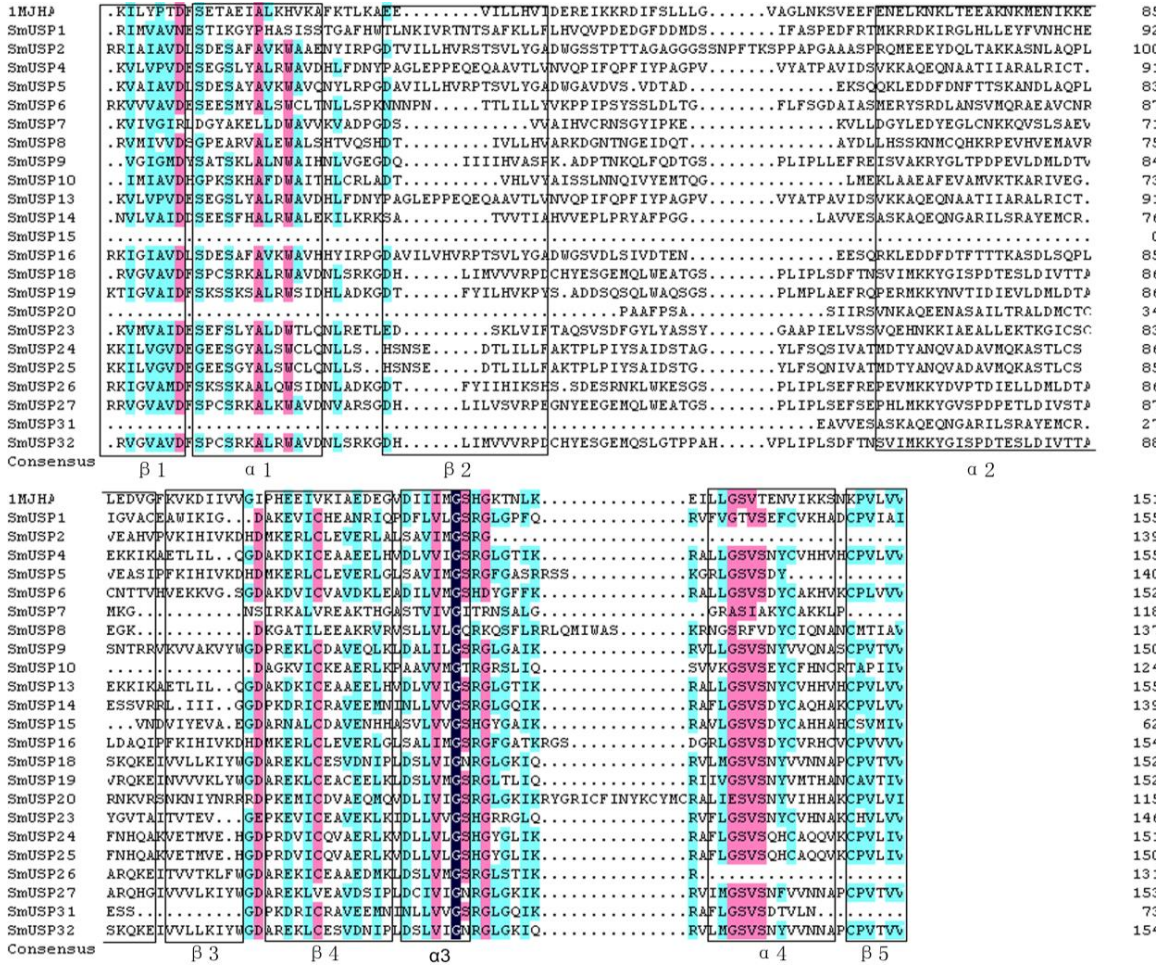

Figure 3. Multiple sequence alignment of 21 SmUSPs and USP MJ0577 (1MJH). The secondary structures involved in ATP-binding function of SmUSPs are marked below sequences according to structure of MJ0577 (1MJH).

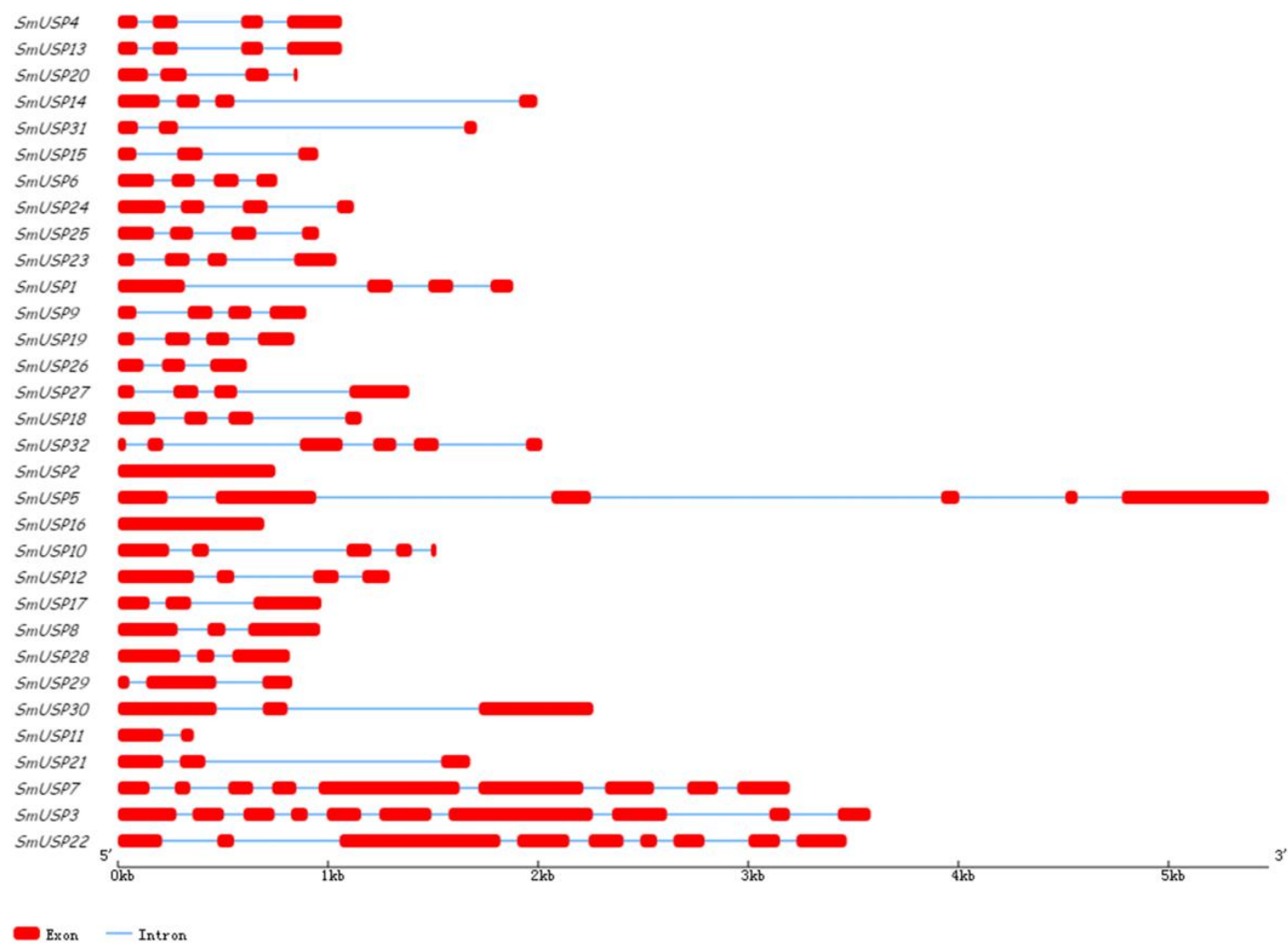

Figure 4. Exon-intron organization structures of 32 SmUSPs. Exons are represented by red, round-cornered rectangles; blue lines connecting 2 exons represent introns. Numbers above lines indicate intron phases. 


\subsection{Patterns of Expression for SmUSP Genes in Various Tissues/Organs}

To profile the expression patterns of SmUSP genes in S. miltiorrhiza, we sampled different tissues and organs for analysis by qRT-PCR (Figure 5). In all, 29 genes were expressed in all five plant parts, while some exhibited diverse profiles. For example, expression was highest in the seeds for 16 SmUSPS and in the roots for 10 genes. Moreover, SmUSP11, SmUSP16, and SmUSP21 showed the highest expression in leaves; SmUSP2 and SmUSP18, in flowers; and SmUSP9, in stems. While SmUSP31 was not expressed in the seeds, nor SmUSP18 in the roots, SmUSP20 was specifically expressed in both stems and seeds.
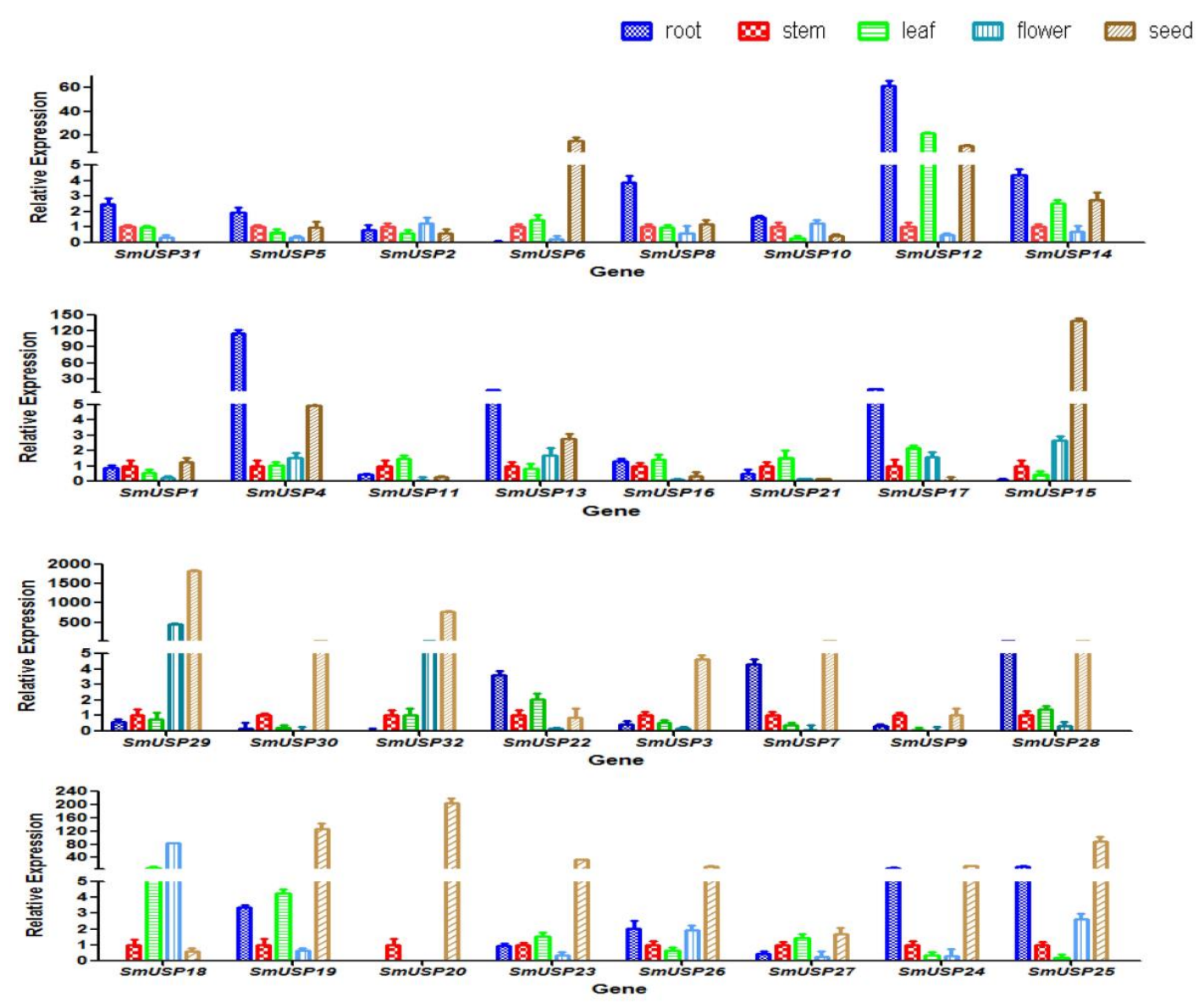

Figure 5. Expression patterns of 32 SmUSP genes in different tissues/organs. Transcription levels were analyzed according to comparative $2^{-\Delta \Delta C(\mathrm{~T})}$ method; all expression levels are relative to that detected in stem samples because all 32 SmUSPs expressed in stem. All data were presented as the means \pm Standard Deviation (SD) of at least three replicates.

3.4. Analysis of Promoter Sequences and Differential Expression of SmUSP Genes in Response to Salt and Heat Stresses and Their Combination

We also investigated the putative stress-responsive cis-elements within the regions of 2-kb genomic sequences upstream of the 5'-UTR of these 32 SmUSPs (Table S4). Among them, 18 contained the ABRE responsible for responses to salinity, dehydration, heat, and cold; 20 had the HSE element involved in heat stress-responsiveness; and 22 carried the MBS element that is a transcription factor MYB binding site involved in drought-inducibility.

Expression pattern of different SmUSP genes were shown in response to either single or combinatorial stress (Figure 6). Our qRT-PCR result showed that 14 SmUSPs (SmUSP1, 2, 4, 7, 8, $10,11,12,13,14,16,17,18,21)$ were significantly induced after salt stress, while 8 (SmUPS6, 15, 19, 22, $25,28,30,32)$ were significantly repressed. For the heat treatment, 13 SmUSPs (SmUSP3, 9, 12, 15, 18, 
$19,23,24,26,27,29,31,32$ ) were significantly upregulated, while 11 (SmUSP2, 5, 6, 8, 10, 11, 13, 16, 21 , 25,30 ) were significantly downregulated. After the salt/heat combination treatment, most SmUSP genes were significantly downregulated, while SmUSP24, 28 were exclusively upregulated.
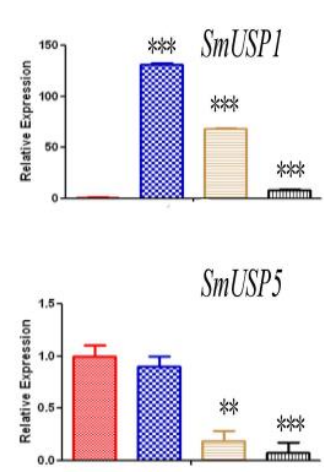

SmUSP9
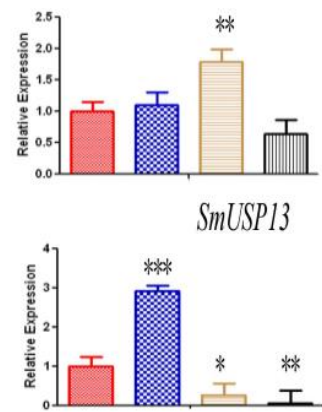

* SmUSJP17

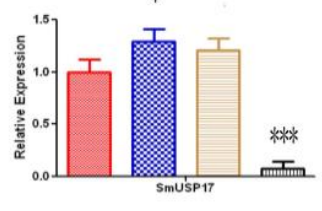

SmUSP22
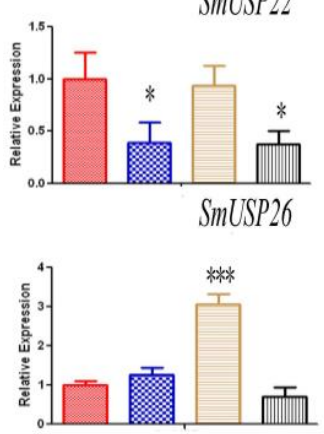

SmLSP30

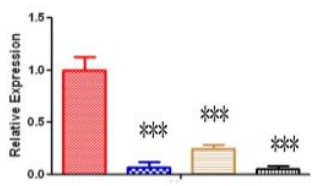

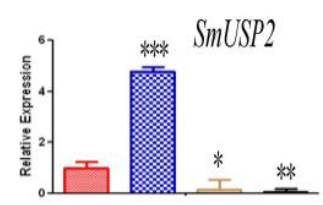
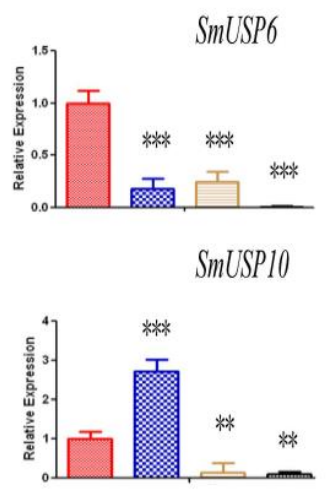

SmLSP14

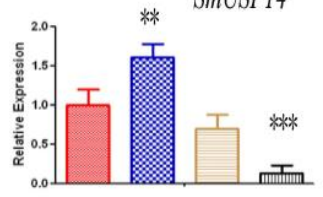

SmUSP18
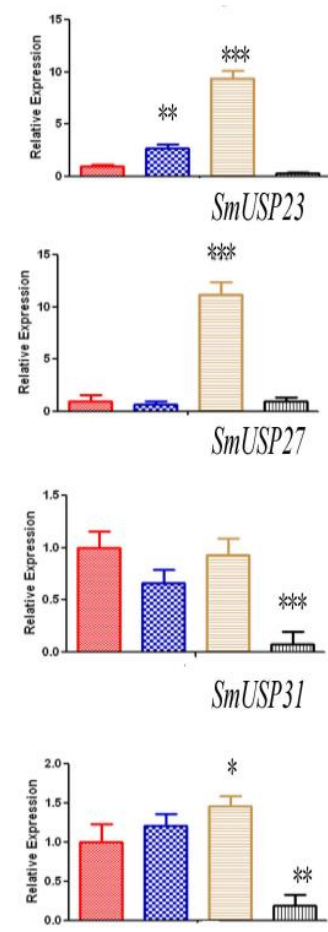
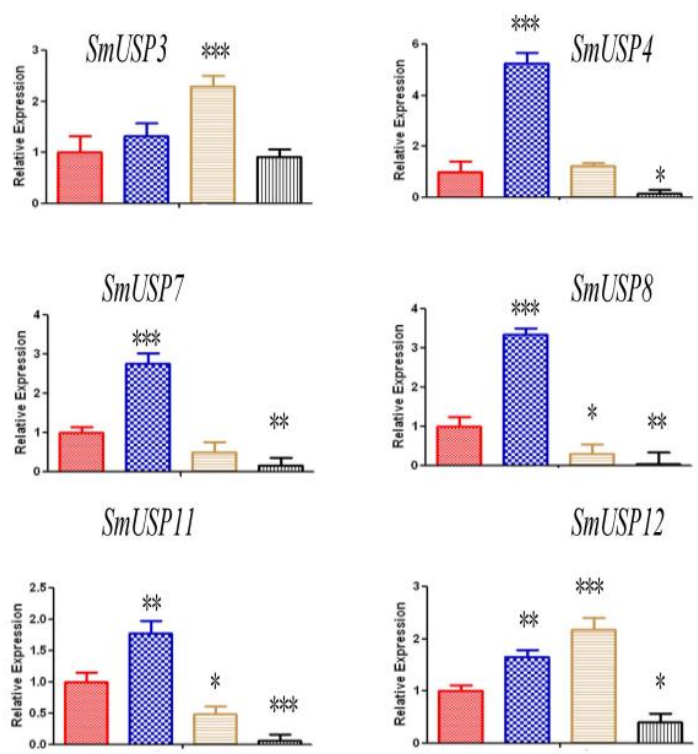

SmUSP15

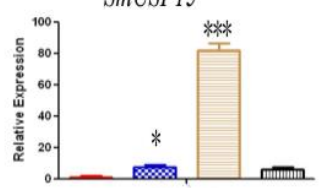

SmUSP19
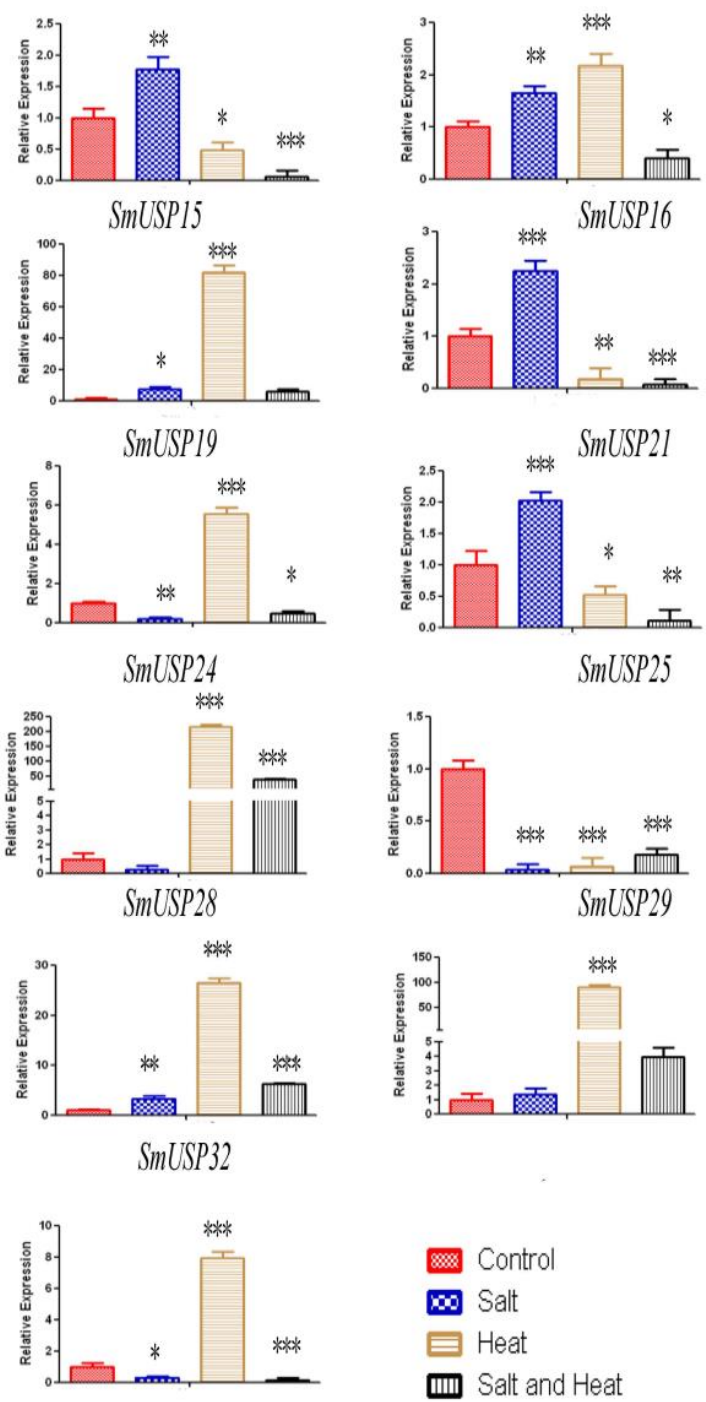

Figure 6. Expression of 32 SmUSP genes under different stress conditions. Based on comparative $2^{-\Delta \Delta C(T)}$ method, all levels are relative to control. For each gene, ${ }^{*}, * *$, and ${ }^{* * *}$ indicate that expression is significantly different from control at $p<0.5,0.1$, and 0.01 , respectively. All data were presented as the means \pm Standard Deviation (SD) of at least three replicates. 


\subsection{Heterologous Expression and Functional Validation in E. coli}

Transformation of pET28a-SmUSP1, pET28a-SmUSP8, and pET28a-SmUSP27 into E. coli BL21 produced protein profiles showing overexpression of approximately $27 \mathrm{kDa}, 30 \mathrm{kDa}$, and $24 \mathrm{kDa}$ recombinant protein, respectively, which was approximately $3 \mathrm{kDa}$ larger than their predicted values (Figure 7).

The effect of SmUSP expression on stress tolerance was studied with E. coli cells. In the spot assay, the number of bacterial colonies expressing each of three genes was compared with that of the control (empty-vector) cells (Figure 8). Under salt, heat, or combined-stress treatment, more colonies containing the constructs expressed SmUSP1, SmUSP8, or SmUSP27 than did the control, indicating that all of these selected genes were able to enhance stress tolerance in that organism. In particular, cells expressing SmUSP27 showed greater tolerance to salt and to the combination treatment when compared with cells expressing SmUSP1 or SmUSP8. In contrast, the level of tolerance to heat stress was strongest in cells expressing SmUSP8.

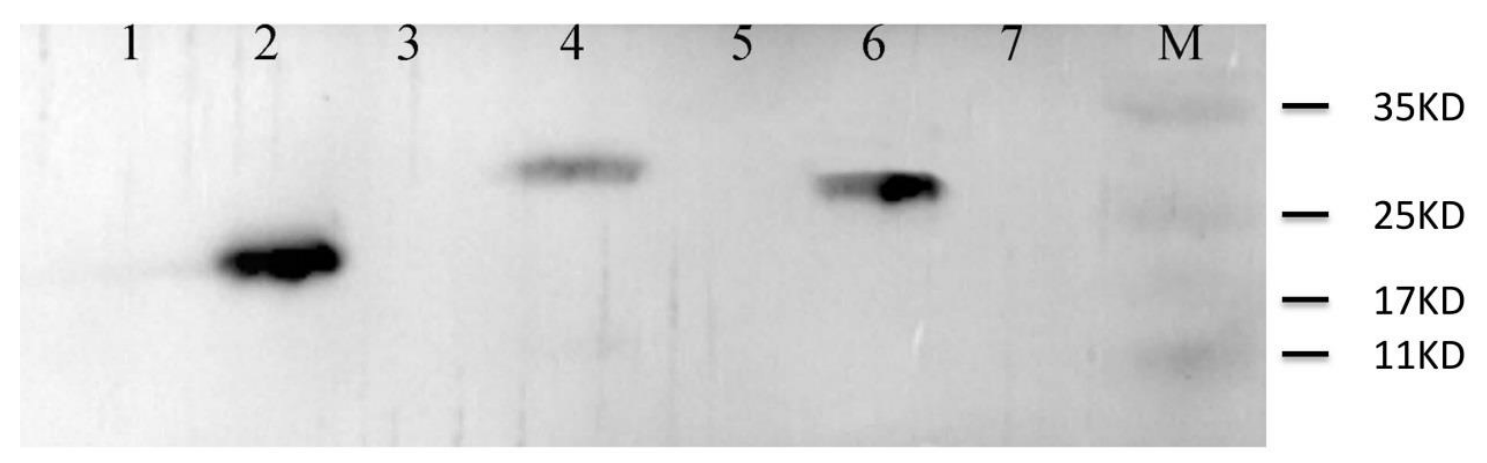

Figure 7. Western blot analysis of SmUSP-overexpression in E. coli BL21. Lanes 1, 3, 5: E. coli BL21 strain harboring pET28a-SmUSP27, pET28a-SmUSP8, and pET28a-SmUSP1 without induction, respectively. Lanes 2, 4, 6: E. coli BL21 strain harboring pET28a-SmUSP27, pET28a-SmUSP8, and pET28a-SmUSP1 induced for $6 \mathrm{~h}$, respectively. Lane 7: E. coli BL21 strain harboring empty pET28a induced for $6 \mathrm{~h}$. M: marker.

For the liquid culture assay, we measured absorbance $\left(\mathrm{OD}_{600 \mathrm{~nm}}\right)$, which reflected the extent of bacterial growth (Figure 9). Under stress conditions, the activity of E. coli cells expressing SmUSP genes increased significantly over time when compared with the control. Salt treatment caused absorbance values for cells harboring SmUSP1, SmUSP8, and SmUSP27 to rise by 2.8-, 2.0-, and 3.7-fold, respectively, over control levels when cells were cultured for $12 \mathrm{~h}$. After heat treatment was applied, absorbance was 1.9-, 3.2-, and 2.8-fold higher, respectively, than in the control samples, while those increases were 1.4-, 1.9-, and 2.0-fold higher, respectively, than those calculated for the control when cells were exposed to the combined salt/heat treatment. Therefore, the resulting trends were consistent between the spot and liquid culture assays. 


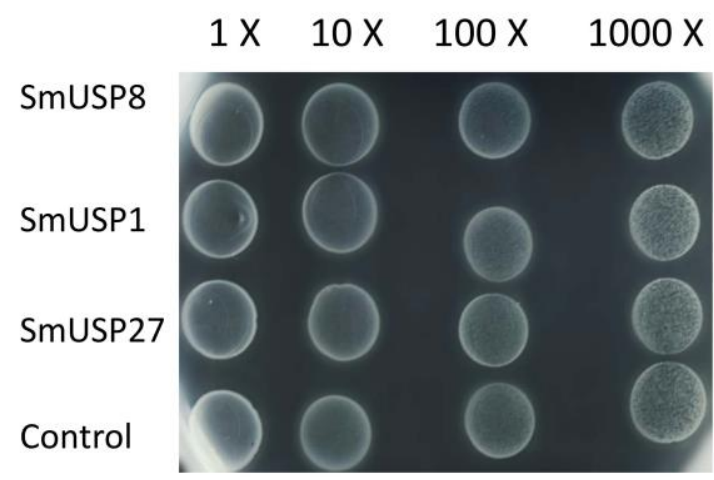

LB
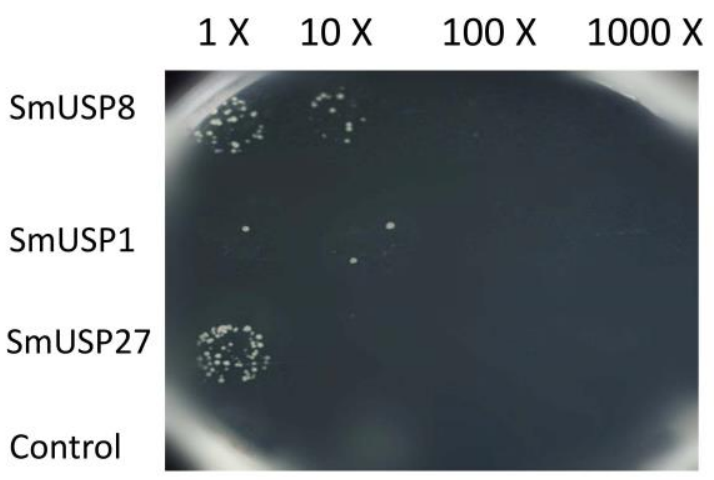

60 min Heat

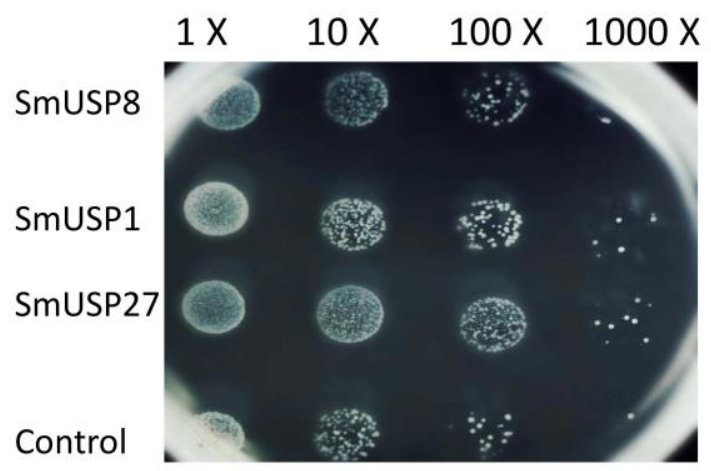

$200 \mathrm{mM} \mathrm{Nacl}$

$1 X \quad 10 X \quad 100 x \quad 1000 X$

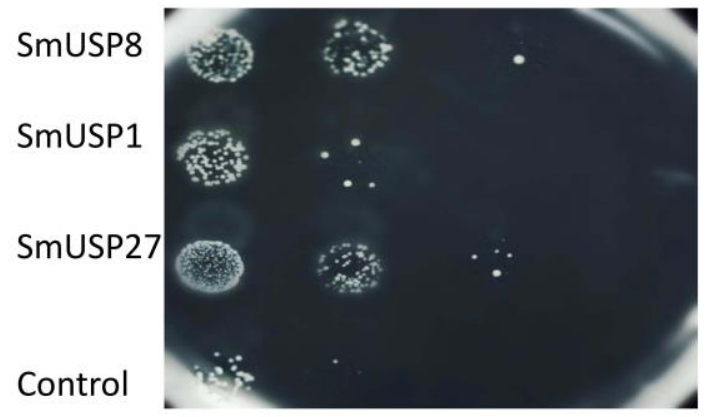

$200 \mathrm{mM} \mathrm{Nacl}$ and $30 \mathrm{~min}$ Heat

Figure 8. Overexpession of SmUSP1, 8, 27 in E. coli enhanced the tolerance to different stresses by spot assay. Each sample was diluted 10-fold, 100-fold, and 1000-fold and spotted onto Luria-Bertani (LB) plates to assess tolerance to various stress treatments. LB represents E. coli BL21 strains transformed plasmids pET28a-SmUSPs and the empty vector pET28a were spotted onto LB solid media; $200 \mathrm{mM}$ $\mathrm{NaCl}$ represents those strains were spotted onto LB solid media containing $200 \mathrm{mM} \mathrm{NaCl}$; 60 min Heat represents those strains were exposed to $50{ }^{\circ} \mathrm{C}$ for $60 \mathrm{~min}$ before $7-\mu \mathrm{L}$ aliquots were spotted on LB media; $200 \mathrm{mM} \mathrm{NaCl}$ and $30 \mathrm{~min}$ Heat represents those strains exposed to $50{ }^{\circ} \mathrm{C}$ for $30 \mathrm{~min}$ before $7-\mu \mathrm{L}$ aliquots were spotted on LB media containing $200 \mathrm{mM} \mathrm{NaCl}$.

LB

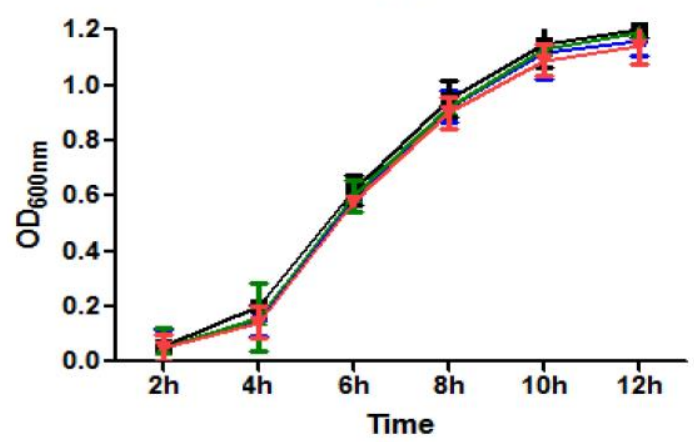

$200 \mathrm{mM} \mathrm{Nacl}$

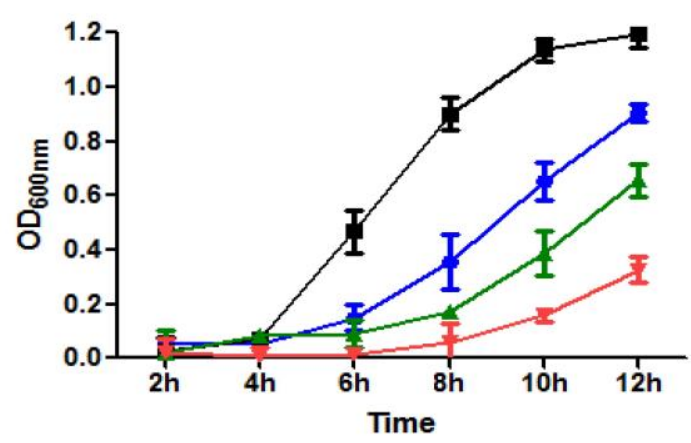

Figure 9. Cont. 
30 min Heat
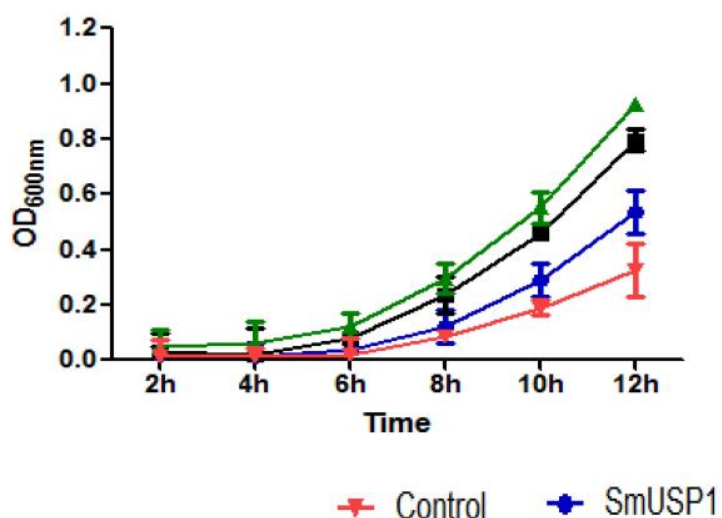

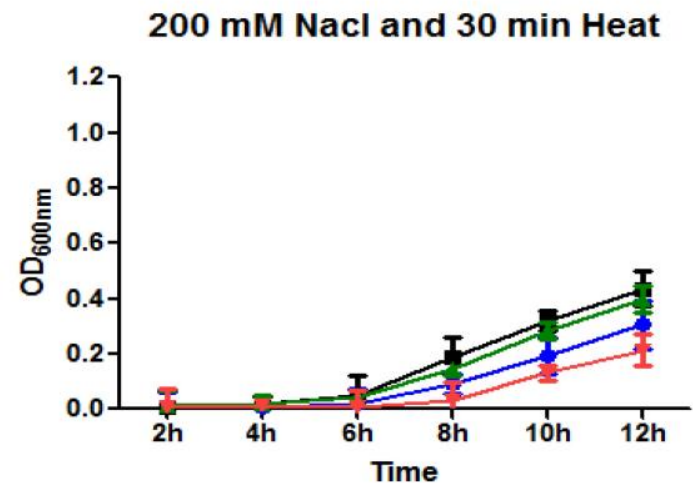

SmUSP8
SmUSP27

Figure 9. Overexpession of SmUSP1, 8, 27 in E. coli enhanced the tolerance to different stresses by liquid assay. Each sample was diluted 200 -fold, using fresh LB media, and absorbance $\left(\mathrm{OD}_{600 \mathrm{~nm}}\right)$ was measured for $12 \mathrm{~h}$ at 2 -h intervals. Each sample has three biological replicates. LB represents samples with the same $\mathrm{OD}_{600}$ value were inoculated into an LB liquid medium (1:200 ratio); $200 \mathrm{mM} \mathrm{NaCl}$ represents those samples were inoculated into an LB liquid medium (1:200 ratio) containing $200 \mathrm{mM}$ $\mathrm{NaCl} ; 30 \mathrm{~min}$ Heat represents those samples were exposed to $50{ }^{\circ} \mathrm{C}$ for $30 \mathrm{~min}$ before being inoculated into an LB liquid medium (1:200 ratio); $200 \mathrm{mM} \mathrm{NaCl}$ and $30 \mathrm{~min}$ Heat represents those samples were exposed to $50{ }^{\circ} \mathrm{C}$ for $30 \mathrm{~min}$ before being inoculated into an LB liquid medium (1:200 ratio) containing $200 \mathrm{mM} \mathrm{NaCl}$.

\section{Discussion}

\subsection{Members of the SmUSP Gene Family and Their Evolution}

Our study is the first to report the identification and characterization of USPs based on the entire genome sequence of $S$. miltiorrhiza. We identified 32 universal stress proteins in S. miltiorrhiza. A search of the NCBI database showed that SmUSP3, 7, and 22 (belonging to subfamily D) contain both an UspA domain and a PKinase domain, while the others have only the UspA domain, similar to what is found with $A$. thaliana [6]. Our phylogenetic analysis led us to organize the 32 SmUSPS into four subfamilies, A through D. The two UspA classes of bacteria include one that binds ATP, i.e., M. jannaschii MJ0577 (1MJH) and one that does not, i.e., Haemophilus influenzae universal stress protein (1JMV) [6]. Further analysis revealed that Motif 1 in Salvia contains the ATP-binding domain and appears in subfamilies A and B, where members belong to the ATP-binding class. The other SmUSPs belong to the non-ATP-binding class. Based on their exon-intron organizational structures, these 32 SmUSP genes are relatively well-conserved. Results from those examinations plus the phylogenetic analysis and multiple sequence alignment suggest that the family members have a common ancestor and have diverged over time.

\subsection{Variations in Gene Expression Patterns}

In S. miltiorrhiza, most of the SmUSPs displayed ubiquitous but highly variable expression in all tissues/organs studied, which suggested functional divergence. Because the 29 genes were detected in all plant parts, it demonstrates that members of this gene family are widely distributed and expressed in that species, and are undoubtedly involved in normal plant growth and development. They include 16 SmUSPs that showed the highest expression in seeds. An Arabidopsis USP, At3g53990 (subfamily A), was widely expressed in most tissues, including the root, stem, leaf, and flower, and was most strongly expressed in the stem. This Arabidopsis USP exhibits a chaperone function, and is induced significantly by heat, $\mathrm{H}_{2} \mathrm{O}_{2}$, and drought [19]. SmUSP9, similar in sequence to At3g53990, showed the highest expression in stems and was induced significantly by heat. Another Arabidopsis USP, At3g62550 
(subfamily A), encodes an ATP-binding motif and is up-regulated in a drought microarray dataset [17]. An SpUSP gene from Solanum pennellii is highly expressed in leaves and stomata, and can reduce the stomatal aperture to protect plants from drought [10]. Both SmUSP11 and SmUSP21 (100\% bootstrap values between them; Figure 1) showed the highest expression in leaves. Their promoter sequences contain a cis-element involved in circadian control, similar to one found in an SpUSP gene (Table S4). These results imply that SmUSP11 and SmUSP21 can reduce stomatal apertures to protect plants of S. miltiorrhiza against the effects of drought; both were induced significantly by heat and drought. SmUSP10, with high sequence similarity to $S L R d 2$, both of which are strongly expressed in roots, might enable S. miltiorrhiza to resist oxidative stress [31]. Thus, our findings that most of these genes are induced by salt, heat, or a combination of the two, is consistent with the results from our analysis of their promoter sequences (Figure 6 and Table S4).

\subsection{SmUSP Genes Enhance Stress Tolerance in E. coli}

The heterologous expression of a USP gene from Salicornia brachiata in E. coli enhances tolerance to salt and osmotic stresses [25]. We selected SmUSP1, SmUSP8, and SmUSP27 because of their heterologous expression in E. coli. All three were expressed in the five tissue/organ samples, with SmUSP1 and SmUSP27 belonging to the ATP-binding class and SmUSP8 belonging to the non-ATPbinding class. Our results showed that, although SmUSP1/8/27 responded differentially to stress conditions, their related proteins conferred tolerance to salt, heat, and the combination treatments when applied to E. coli. Compared to SmUSP8, SmUSP27 and SmUSP1 conferred greater tolerance to salinity in that organism while SmUSP8 conferred the highest tolerance to heat stress. This was probably because the two separate classes of USP exhibit different functions in S. miltiorrhiza. For example, whereas SmUSP27 induced the highest tolerance against both salt stress and the combination treatment in E. coli, transcription profiling indicated that SmUSP8 was significantly up-regulated only by heat stress. Results from previous studies have also demonstrated that the response of a plant to a combination of two or more stress conditions is unique and one cannot directly extrapolate this to the response a plant might have to those stresses when applied individually [5].

\subsection{Function of SmUSPs under Simultaneous Stresses}

In contrast to having controlled growth conditions when experiments are conducted in the laboratory, a field environment often involves the simultaneous exposure of plants to more than one type of abiotic and/or biotic stress [5]. Our results indicated that the three selected SmUSPs are less tolerant to a combined stress than to salt or heat stress alone. Among the 32 SmUSP genes, 24 were significantly induced by the combined treatment while 22 and 25 were significantly induced by salt and heat stress, respectively (Figure 6). This led to a high degree of complexity in plant responses that are largely controlled by different, and sometimes opposing, signaling pathways that may interact and inhibit each other [4]. For example, in a study of Triticum aestivum, researchers found that the combined effects of drought plus heat on grain yields were greater than the influence that those stressors had individually [32]. In contrast, tomato plants were much better protected against the combination of salt and heat stresses than they were when challenged with salinity alone [33]. Our data demonstrate how important it is that we develop new plant lines with enhanced tolerance to various stress combinations. With that goal, we propose that future investigations into the functions of all SmUSPs should also be validated in both E. coli and S. miltiorrhiza.

Supplementary Materials: The following are available online at www.mdpi.com/2073-4425/8/9/224/s1, Figure S1: Motif 2-10 of SmUSP proteins, Table S1: Primers for qRT-PCR, Table S2: Primers for cloning the full-length of 3 SmUSPs, Table S3: The characteristics of the 32 SmUSP proteins, Table S4: Promoter sequences analysis of 32 SmUSPs.

Acknowledgments: We are very grateful to two anonymous reviewers for their helpful suggestions and comments. This research was supported by the National Natural Science Foundation of China (31670299), the major Project of Shaanxi Province, China (2017ZDXM-SF-005) and the Fundamental Research Funds for the Central Universities (GK201706004). 
Author Contributions: X.-Y.C. and J.-F.K. designed the experiments and wrote the paper. X.-F.W., J.S. and N.Y. performed the experiments. X.-F.W. and H.Z. analyzed the data.

Conflicts of Interest: The authors declare that they have no conflict of interest.

\section{References}

1. Serrano, R.; Rodriguez-Navarro, A. Ion homeostasis during salt stress in plants. Curr. Opin. Cell Biol. 2001, 13, 399-404. [CrossRef]

2. Zhu, J.K. Plant salt tolerance. Trends Plant Sci. 2001, 6, 66-71. [CrossRef]

3. Heremans, K.; Smeller, L. Protein structure and dynamics at high pressure. Biochim. Biophys. Acta 1998, 1386, 353-370. [CrossRef]

4. Howarth, C.J. Genetic Improvements of Tolerance to High Temperature; Howarth Press: Philadelphia, PA, USA, 2005.

5. Suzuki, N.; Rivero, R.M.; Shulaev, V.; Blumwald, E.; Mittler, R. Abiotic and biotic stress combinations. New Phytol. 2014, 203, 32-43. [CrossRef] [PubMed]

6. Kerk, D.; Bulgrien, J.; Smith, D.W.; Gribskov, M. Arabidopsis proteins containing similarity to the universal stress protein domain of bacteria. Plant Physiol. 2003, 131, 1209-1219. [CrossRef] [PubMed]

7. Ndimba, B.K.; Chivasa, S.; Simon, W.J.; Slabas, A.R. Identification of Arabidopsis salt and osmotic stress responsive proteins using two-dimensional difference gel electrophoresis and mass spectrometry. Proteomics 2005, 5, 4185-4196. [CrossRef] [PubMed]

8. Persson, O.; Valadi, A.; Nyström, T.; Farewell, A. Metabolic control of the Escherichia coli universal stress protein response through fructose-6-phosphate. Mol. Microbiol. 2007, 65, 968-978. [CrossRef] [PubMed]

9. Kvint, K.; Nachin, L.; Diez, A.; Nyström, T. The bacterial universal stress protein: Function and regulation. Curr. Opin. Microbiol. 2003, 6, 140-145. [CrossRef]

10. Rachid, L.; Wang, T.; Bo, O.; Khurram, Z.; Li, H.; Zhang, J. SpUSP, an annexin-interacting universal stress protein, enhances drought tolerance in tomato. J. Exp. Bot. 2012, 63, 5593-5606.

11. Li, W.T.; Wei, Y.M.; Wang, J.R.; Liu, C.J.; Lan, X.J.; Jiang, Q.T. Identification, localization, and characterization of putative USP genes in barley. Theor. Appl. Genet. 2010, 121, 907-917. [CrossRef] [PubMed]

12. Tkaczuk, K.L.; Shumilin, I.A.; Chruszcz, M.; Evdokimova, E.; Savchenko, A.; Minor, W. Structural and functional insight into the universal stress protein family. Evolut. Appl. 2013, 6, 434-449. [CrossRef] [PubMed]

13. Sauter, M.; Rzewuski, G.; Marwedel, T.; Lorbiecke, R.A. The novel ethylene-regulated gene OsUsp1 from rice encodes a member of a plant protein family related to prokaryotic universal stress proteins. J. Exp. Bot. 2002, 53, 2325-2331. [CrossRef] [PubMed]

14. Maqbool, A.; Zahur, M.; Husnain, T.; Riazuddin, S. GUSP1 and GUSP2, two drought-responsive genes in Gossypium arboreum have homology to Universal Stress Proteins. Plant Mol. Biol. Rep. 2009, 27, 109-114. [CrossRef]

15. Chou, M.X.; Wei, X.Y.; Chen, D.S.; Zhou, J.C. A novel nodule enhanced gene encoding a putative universal stress protein from Astragalus sinicus. J. Plant Physiol. 2007, 164, 764-772. [CrossRef] [PubMed]

16. Becker, J.D.; Moreira, L.M.; Kapp, D. The nodulin vfENOD18 is an ATP-binding protein in infected cells of Vicia faba L. nodules. Plant Mol. Biol. 2001, 47, 749-759. [CrossRef]

17. Isokpehi, R.D.; Simmons, S.S.; Cohly, H.H.P.; Ekunwe, S.I.N.; Begonia, G.B.; Ayensu, W.K. identification of drought-responsive universal stress proteins in Viridiplantae. Bioinform. Biol. Insights 2012, 5, 41-58. [CrossRef] [PubMed]

18. Zahur, M.; Maqbool, A.; Irfan, M.; Barozai, M.Y.K.; Rashid, B.; Riazuddin, S. Isolation and functional analysis of cotton universal stress protein promoter in response to phytohormones and abiotic stresses. Mol. Biol. 2009, 43, 578-585. [CrossRef]

19. Jung, Y.J.; Melencion, S.M.B.; Lee, E.S.; Park, J.H.; Alinapon, C.V.; Oh, H.T. universal stress protein exhibits a redox-dependent chaperone function in arabidopsis and enhances plant tolerance to heat shock and oxidative stress. Front. Plant Sci. 2015, 6, 1141. [CrossRef] [PubMed]

20. Wang, J.W.; Wu, J.Y. Tanshinone biosynthesis in Salvia miltiorrhiza and production in plant tissue cultures. Appl. Microbiol. Biotechnol. 2010, 88, 437-449. [CrossRef] [PubMed] 
21. Hui, X.; Lin, Z.; Zhou, C.C.; Xiao, J.B.; Pan, L.; Kai, G.Y. Metabolic regulation and genetic engineering of pharmaceutical component tanshinone biosynthesis in Salvia miltiorrhiza. J. Med. Plant Res. 2011, 4, 2591-2597. [CrossRef]

22. Dong, Y.; Morris-Natschke, S.L.; Lee, K.H. Biosynthesis, total syntheses, and antitumor activity of tanshinones and their analogs as potential therapeutic agents. Nat. Prod. Rep. 2011, 28, 529. [CrossRef] [PubMed]

23. Li, C.; Lu, S. Genome-wide characterization and comparative analysis of R2R3-MYB transcription factors shows the complexity of MYB-associated regulatory networks in Salvia miltiorrhiza. BMC Genom. 2014, 15, 277. [CrossRef] [PubMed]

24. Xu, H.B.; Song, J.Y.; Luo, H.M.; Chen, S.L. Analysis of the genome sequence of the medicinal plant Salvia miltiorrhiza. Mol. Plant 2016, 9, 949. [CrossRef] [PubMed]

25. Udawat, P.; Mishra, A.; Jha, B. Heterologous expression of an uncharacterized universal stress protein gene (SbUSP) from the extreme halophyte, Salicornia brachiata, which confers salt and osmotic tolerance to E. coli. Gene 2014, 536, 163. [CrossRef] [PubMed]

26. Xu, Z.C.; Ji, A.J.; Song, J.Y.; Chen, S.L. Genome-wide analysis of auxin response factor gene family members in medicinal model plant Salvia miltiorrhiza. Biol. Open. 2016, 5, 848-857. [CrossRef] [PubMed]

27. Schmittgen, T.D.; Livak, K.J. Analyzing real-time PCR data by the comparative C(T) method. Nat. Protoc. 2008, 3, 1101. [CrossRef] [PubMed]

28. O'Toole, R.; Williams, H.D. Universal stress proteins and Mycobacterium tuberculosis. Res. Microbiol. 2003, 154, 387-392. [CrossRef]

29. Gilbert, W. The exon theory of genes. Cold Spring Harb. Symp. Quant. Biol. 1987, 52, 901. [CrossRef] [PubMed]

30. Patthy, L. Intron-dependent evolution: Preferred types of exons and introns. FEBS Lett. 1987, $214,1-7$. [CrossRef]

31. Gutiérrez-Beltrán, E.; Personat, J.M.; de la Torre, F.; del Pozo, O. A universal stress protein involved in oxidative stress is a phosphorylation target for protein kinase CIPK6. Plant Physiol. 2017, 173, 836-852. [CrossRef] [PubMed]

32. Pvv, P.; Pisipati, S.R.; Momčilović, I.; Ristic, Z. Independent and combined effects of high temperature and drought stress during grain filling on plant yield and chloroplast EF-Tu expression in spring wheat. J. Agron. Crop Sci. 2012, 197, 430-441.

33. Rivero, R.M.; Mestre, T.C.; Mittler, R.; Rubio, F.; Garcia Sanchez, F.; Martinez, V. The combined effect of salinity and heat reveals a specific physiological, biochemical and molecular response in tomato plants. Plant Cell Environ. 2014, 37, 1059. [CrossRef] [PubMed] 